\title{
The Potential for Individualization of Neoadjuvant Chemotherapy in Breast Cancer
}

\author{
Hojouj Mohammad IM* \\ Department of Oncology and Medical Radiology, Ukraine \\ *Corresponding author: Hojouj Mohammad IM, Department of Oncology and Medical Radiology, VVernadskiiStreet, 9, Dnipro, 49044, Ukraine
}

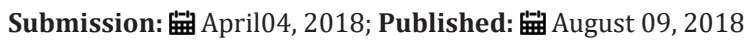

\section{Introduction}

The preoperative, or, as it is often called, neoadjuvant chemotherapy (NAPCT) of operable breast cancer (the breast cancer) with affected regional lymph nodes has in its time replaced preoperative radiotherapy, as it has a number of significant advantages over the latter. The most important advantage of NAPCT is its systemic action, which allows to "catching up" with probable distant micro-metastases or circulating tumor cells, while pre-operative radiotherapy has a local effect, and on the systemic level the tumor continues to develop. Despite of the fact that with operative breast cancer the time of NAPCT (before or after the operation) does not affect to the long-term results of treatment, the latter becomes applicable even for operable breast cancer without affected regional lymph nodes.According to the literature, NAPCT with primary-operative breast cancer allows: 1) make organ saving operations; 2 ) improve the prognosis in cases of complete morphological regression in patients with triple negative and Her2 / neu positive (non-luminal) subtypes; 3) evaluate the effect of chemotherapy and, in the absence of effect, stop it on time [1].

It should be noted that 292 clinical studies on the latest data of the site https://clinicaltrials.gov/ are devoted to the study of the effectiveness and significance of neoadjuvant therapy of the breast cancer. The schemes that are studied mainly include rather familiar medicines like taxanes (docetaxel, paclitaxel, nab-paclitaxel), anthracyclines (doxorubicin, epirubicin), platinum derivatives (mainly carboplatin), alkylating agents (cyclophosphamide), fluoropyrimidines (fluorouracilS-1), antimetabolites (gemcitabine), vinaalkaloids (vinorelbine), hormonal agents (aromatase inhibitors, antiestrogens), and others. Also are used targeted medicines (trastuzumab), inhibitors of angiogenesis (bevacizumab), immune preparations (atezolizuma b), etc. In some studies we can see using of modifiers of the action of hormonal agents or chemotherapeutics (metformin, celecoxib, L-carnitine, etc.). Also, new medicines (Myocet, AC4-CDDP4, R04929097, HM10460A) and medicines that are commonly used to treat or correct non-tumor diseases and conditions (Atorvastatin, CoenzymeQ10, Melatonin, aspirin) or phytopreparations and biologically active additives (Reishimushroomextract, Yunzhiextract) are investigated [2].
It has been shown that the breast cancer is sensitive to the most anti-tumour medicines, especially to doxorubicin and epirubicin (efficacy in $40-50 \%$ of patients with the metastatic breast cancer), methotrexate (efficacy in 35\% of patients), fluorouracil and tegafur (efficacy in $25-34 \%$ of patients). Better efficacy is indicated by "newer" drugs. Thus, paclitaxel is effective in 32-62\% of patients, docetaxel in $50-75 \%$, vinorelbin in $41-51 \%$, topotecan in $36 \%$, mitoxantrone in $20-35 \%$ of patients with the metastatic breast cancer in the first line [3]. In patients with overexpression of Her2 / neu a significant effect has Herceptin. In patients with Her2 / neu positive breast cancer its using in combination with sequential anthracycline and taksane-containing chemotherapy allows complete pathomorphological regression in more than $62 \%$ of patients compared with $26 \%$ without trastuzumab [4,5].

For using in the neoadjuvant regimen NCCN primarily recommends compactedozorozhimas AC (doxorubicin+cyclophosphamide) with sequential using of paclitaxel or TC (docetaxel in combination with cyclophosphamide). Others recommended modes are compacted AC, CMF, EC, TAC. In the case of Her2/neu positive tumor it is recommended to administer paclitaxel with trastuzumab or paclitaxel with trastuzumab and pertuzumab first after the AS regimen. Other variants of the priority regimens are TSN (docetaxel+carboplatin+herceptin) or TSN + pertuzumab [6]. The using of priority regimes is very costly. Treatment of complications (most often neutropenia of grade 3-4 NCNCTCAE or febrile neutropenia) requires considerable additional funds. Supportive therapy with colony-stimulating factors is not much cheaper. With good funding the recommended regimens are acceptable. However, with limited financial support, the implementation of these recommendations is almost impossible. This factor leads to the appointment of less effective regimens of treatment, which leads to a deterioration of the results of treatment.

Also, misdiagnosis of primary treatment can lead to the development of acquired resistance to the subsequent treatment line. It can significantly complicate the choice of the next regime and significantly increase its cost, while dramatically reducing its effectiveness.Therefore, the definition of optimal therapy is the 
most important question that must be answered before starting treatment. Modern possibilities of laboratory diagnostics allow to personify the therapeutic tactics. For it are used methods to study individual prognosis and the risk of relapse and distant metastases (MammaPrint, Prosigna, OncotypeDX, TCGA). However, even in developed and financially stable countries, the application of these methods may be limited. In countries with limited material capacity its application is unrealistic.Using of modern genetic and other high-tech studies of tumors in Ukraine is inaccessible; we will try to consider approaches to the choice the systemic treatment of the breast cancer, given the possibilities of immunohistochemical diagnosis and the ability to predict response of treatment using immunohistochemical markers.Immunohistochemical study of biological markers of the breast cancer has two main aims: to identify among patients with early stages of the disease the groups of increased risk of tumor progression, which require in additional examination and/or treatment; to assessment of individual sensitivity to therapy, which is planned or carried out [7].

It is known that the molecular classification of the breast cancer is rather complicate. Therefore, for convenience, in practice, the so-called surrogate molecular classification, including luminal subtypes, Her2-enriched, basal and rare types, is used. Among the luminal subtypes are subtype $\mathrm{A}$, which, according to some data constitutes up to $40 \%$ of all breast cancer and is characterized by positive receptors for estrogen (ER), progestins (PR), no overexpression of epidermal growth factor receptors (Her2 / neu) and low proliferation marker Ki67 $(<20 \%)$. The luminal B subtype of the tumor is divided into the so-called Her2-negative and Her2positive. The luminal Her2-negative subtype differs from Luminal A by elevated or high level $(\geq 20 \%)$ of the Ki67 marker. Luminal B Her2-positive subtype expresses Her2 / neu, has a high level of marker Ki67. Cases, when with the low level of proliferation is Her2 / neu expressed (Ki67 <20\%) should be treated separately [8].

The determination of the status of sex hormone receptors and epidermal growth factor is a classic example of molecular individualization of the breast cancer therapy [9]. The predictive value of the determination of EP and PR for endocrine therapy was confirmed by a meta-analysis of 55 randomized trials of 37,000 breast cancer patients. It was proved that the presence of EP in the breast tumor testifies to its potential sensitivity to medical measures that are aimed on removing from the body of the source of estrogens or countering their effects. The presence of PR indicates the functional activity of EP. Patients with the breast cancer whose tumors have both or at least one of the steroid hormone receptors have a more favorable prognosis than those in which these receptors are negative. So the effectiveness of endocrine therapy reaches $75 \%$ with both positive receptors, $50 \%$ with positive only EP. However, $10 \%$ of patients, who's both receptors are negative, are also sensitive to endocrine therapy [7].A high level of estrogen receptors is associated, first of all, with an increase of general survival [10], and is a predictor of the effectiveness of hormone therapy. In recent years, the Ki-67 proliferation index has been used to predict the effectiveness of treatment $[8,11,12]$.
The panel of markers of estrogen, progestin receptors, Her2/ neu and Ki-67, proposed by Cuzick Jet al.[13], is now the standard at the stage of primary diagnosis and during morphological studies during treatment. In view of these four markers, the main immunomorphological subtypes of breast cancer mentioned above are distinguished.In the case of neoadjuvant treatment or treatment of metastatic cancer (with the possibility of repeated biopsy in dynamics), Ki-67 has recently been used as a marker of treatment effectiveness. Repeated biopsy is made 3 weeks after the start of treatment. Reduction of the level of the marker is the first morphological predictor of the effectiveness of treatment [11], not only hormone, but chemotherapy [12]. Conversely, increasing the level of the marker is a predictor of an unfavorable prognosis [14]. The possibilities of immunohistochemical studies in the choice of hormonal therapy for luminal A and Her2/neu negative luminal breast cancer have been reviewed previously [15]. Also, we should consider the possibilities of immunohistochemical studies in the selection of the optimal regimen of chemotherapy. To do this, let us consider which immunohistochemical markers can orient in the selection of individual cytostatics.

Cyclophosphamide the medicine is metabolized with forming the metabolites that have an alkylating effect. Alkylating metabolites attack the nucleophilic centers of protein molecules and block the mitosis of tumor cells. Through the cytochrome system, cyclophosphamide is at first metabolized into 4-hydrocyclophosphamide and its tautomeric form, aldophosphamide. Aldophosphamide with $\beta$-elimination is spontaneously split into acrolein and phosphoramide mustard. Phosphoramide mustard has a major biological effect - alkylates DNA, in resulting of it formation the intra-strand and crosslinking DNA [16].The only immunohistochemical marker of hypersensitivity to cyclophosphamide is TLE3. By the way, the expression of this marker is an indicator of hypersensitivity to taxanes. Thus, their combination is quite effective just in the case of marker expression [17]. At the same time, the expression of detoxifying protein glutatin-S-transferase (GST) causes resistance to alkylating compounds (embichin, chlorbutin, melphalan, cyclophosphamide) and platinum preparations [18]. The detoxification effect is provided by the conjugation of genotoxic metabolites with glutathione, which ensures their inactivation [19]. The expression of another detoxifying protein of the P-glycoprotein (P-gp, MDR1, CD243) affects the efflux (active excretion of the medicine from the cell) and the pharmacodynamics of the main cytostatics that used in clinical oncology: mitoxantrone, topotecan, methotrexate, doxorubicin, daunorubicin, actinomycin D, vinblastine, vincristine, paclitaxel. Cyclophosphamide, which has long been the "gold standard" of the breast cancer treatment for the frequency of complete morphological responses on treatment, is equally effective in both P-gp-negative and P-gp-positive tumors [20].

Methotrexate inhibits dihydrogenphosphate reductase (DHF) converting dihydrofolic acid to tetrahydrofolic acid, which is a donor of single-carbon groups in the synthesis of purine nucleotides and 
thymidylate, which are necessary for DNA synthesis. In the cell methotrexate undergoes polyglutaminization with the formation of metabolites that also inhibit folate-dependent enzymes. It has S-phase specificity, is active against tissue with high proliferative activity of cells, inhibits the growth of malignant neoplasms [21]. There are no specific markers or predictors of high tumor sensitivity to methotrexate. However, there are markers of increased toxicity and resistance. Thus, candidates for methotrexate therapy should study the presence of polymorphism -665C/T, 677T/T and 401C/T of the MTHFR gene, since its presence in tens of times increases the risk of side effects of the drug [22]. The mutation rate is quite high (10-16\%) [23]. At the same time, the administration of folic acid, parallel to methotrexate, significantly reduces its side effects [22]. The study is not costly. The material for the study is whole blood, that is taken with the addition of ethylenediaminetetraacetic acid, and is performed by PCR and restriction analysis. Among the genes that may be associated with resistance to methotrexate, should remember about the gene of retinoblastoma and the gene $\mathrm{p} 53$. The absence of a protein that is encoded by the retinoblastoma gene can lead to resistance to methotrexate due to increased production of DHF as a result of increased translation of its mRNA without amplification of the coding gene [21].

5-fluorouracil, tegafur and capecitabine belong to the group of pyrimidine agonists, which inhibit enzymes, that are necessary for the synthesis of nucleic acids and can be incorporated into DNA and RNA [24].5-fluorouracil disrupts the synthesis of DNA and leads to the formation of structurally deficient RNA, thereby suppressing the division of tumor cells. The mechanism of its action is determined by its metabolic conversion to 5-fluoro-deoxyuridine-monophosphate and 5-fluorouridine tri-phosphate. The first competitively inhibits thymidylate synthetase, which leads to the blocking of DNA synthesis. It blocks the methylation reaction of deoxyuridic acid and its transformation into thymidyl acid, which leads to thymidine deficiency. The second is included in the structure of RNA instead of uridine triphosphate, thus, suppresses its synthesis. This leads to disruption of RNA processing and protein synthesis.Tegafur is metabolized in the liver with the formation of metabolites, among which the leading place is occupied by active 5 -fluorouracil. Tegafur bioactivation is carried out not only in the liver, but can also be local in the tumor tissue, which has an increased content of cytosolic hydrolytic enzymes.Capecitabine is activated in the tumor tissue by changing to 5 -fluorouracil under the action of tumor angiogenic factor thymidine phosphorylase. The mechanism of anticancer conversion of capecitabine to active fluoropyrimidine is associated with increased expression of thymidylate phosphorylase enzyme in tumors. If the level of TP is low, the appointment of capecitabine is impractical, although such tumors are characterized by a better response to classical fluoropyrimidines, in particular to 5-fluorouracil [25]. It has been shown that the increased content of thymidylate synthetase (TS) can prevent the "saturation" of the therapeutic target and correlates with the low efficacy of fluoropyrimidines [26].
Metabolism of fluorouracil is carried out, first of all, in the liver under the action of the enzyme dihydropyrimidine dehydrogenase (DPD), the level of which determines the toxicity of the medicine. Epilepsy is often associated with the decreasing or absencing of the DPD function, therefore, fluoropyrimidines should be given very carefully to patients with this pathology [27]. Anthracyclines (doxorubicin, epirubicin) and their synthetic derivatives (mitoxantrone) inhibit the synthesis of DNA and RNA by intercalation into a double helix of DNA between pairs of nitrogenous bases and cause DNA splitting due to the formation of free radicals. In addition, the antitumor effect is due to changes in cellular functions in result of binding with the lipids of cell membranes and interaction with topoisomerase II [28]. Topoisomerase II $\alpha$ is the target for anthracyclines. High expression and/or co-amplification of TOP2A and Her/2 contribute to better results in anthracycline therapy [29]. At the same time, some mutations of TOR2A are associated with the development of cancer cell resistance in anthracycline chemotherapy [30]. Anthracycline resistance is associated with $\mathrm{ABC}$ transports and p53 mutation. For example, the AB-transporter P-gp responsible for the reverse transport of anthracyclines (including mitoxantrone), vinca alkaloids, taxanes, mitomycin C, topotecan, irinotecan [31], BCRP "squeezes" out of the cell methotrexate, topotecan, mitoxantrone, doxorubicin [32,], and MRP1 removes paclitaxel, methotrexate, anthracyclines, vincaalkaloids and antiandrogens from the cell [33]. The p53 mutation lowers the sensitivity to doxorubicin by a factor of two compared to $\mathrm{p} 53$. Treatment with epirubicin is more effective in patients with mutanted p53. The complete morphological response to neoadjuvant treatment according to the EC scheme in patients with p53 mutation exceeds 50\% [34]. Taxanes (paclitaxel and docetaxel) are represented by two molecules, that are isolated from plants of the Taxus family (yew). Now are drugs with less toxicity than their previous forms (abraxane) have. The toxicity of abraxane is reduced by replacing the stabilizing agent in the pharmaceutical form with cremophor to albumin.

Paclitaxel activates the assembly of microtubules from tubulin dimers and stabilizes them, preventing depolymerization. As a result, the dynamic reorganization of the microtubule network in the interphase and during mitosis is inhibited. Docetaxel also damages the microtubular network in cells at the stage of mitosis and in interphase. It is combined with free tubulin, stimulates the accumulation of tubulin in stable microtubules and prevents their disintegration. As a result, microtubule bonds are formed, which are stabilized, and are losed their ability to function normally, which leads to a disruption of the phase of mitosis and interphase interactions in cells [35]. The marker of sensitivity of the breast cancer to taxanes is beta-tubulin III grade. However, the results of various studies on the significance of this marker are contradictory. High level of expression of beta-tubulin III class in combination with negative estrogen receptors determines a group of patients with the breast cancer with increasing sensitivity to chemotherapy regimens that are containing taxanes, anthracyclines and cyclophosphamide [36]. 
At the same time, a number of researchers consider high expression of beta-tubulin III class as an extremely unfavorable prognosis factor associated with a decrease of patient survival [37]. Other authors consider the level of beta-tubulin III as a marker of tumor prevalence, but do not consider it like the predictor of treatment [38]. Some scientists consider the low level of marker expression as an indicator of hypersensitivity to treatment regimens that are based on taxanes [39]. The author also believes that the effect of docetaxel treatment is better in patients with a p53 mutation.Paclitaxel also induces apoptosis by phosphorylation of the anti-apoptotic Bcl-2 gene, enhancing the regulation of the proapoptotic Bak gene and activating c-JunNH2-terminalkinase [40]. Therefore, expression of Bcl-2 is also one of the prognostic markers of sensitivity to paclitaxel.

Vinca alkaloids and platinum preparations are mainly used in the treatment of metastatic breast cancer [41]. In non-adjuvant regimens, these drugs can only be used as an exception for purposes of rigid personification. However, it makes sense to consider and the possibility of their use in preoperative treatment.Vinca alkaloids (vincristine, vinblastine, vinflubin, vinorelbine), like and taxanes, affect on the microtubules. They bind with the tubulin, inhibit the formation of the mitotic spindle; stop the mitotic division of cells at the metaphase stage. In high doses, also, they inhibit the synthesis of nucleic acids and protein [42]. There are no specific markers responsible for the hypersensitivity of the tumor to vinca alkaloids. There are certain parallels of the sensitivity of vincaalkaloids with a similar sensitivity mechanism for taxanes, which are associated with Class III beta tubulin $[38,43]$.
For the resistance of treatment by vinaalkaloids are responsible the P-gp (MDR1) [32,44] BRCA mutations [45] and Breast cancer resistance protein (BCRP / ABCG2) [46]. Moreover, vincristine itself can become an inducer of P-gp and MRPs (Multidrug resistance associated proteins) [47]. Cisplatin bifunctionally alkylates DNA strands, inhibits the biosynthesis of nucleic acids and causes cell death. At the first stage it inhibits the synthesis of DNA, RNA and protein, and on the second it forms metabolic products that act on the synthesis of DNA. Platinum complexes with cis-arrangement of halogen atoms form stable chelates with purine and pyrimidine components of the nucleic acid molecule and form bonds within one filament or parallel strands of a double helix of DNA. The blockage of DNA strands persists for several days after cisplatin administration [48]. The mutation of the BRCA1 gene is associated with a high sensitivity of the tumor to platinum [49]. The absence or the low level of expression of signals of system's repair of DNA ERCP- 1 also indicates a high sensitivity to platinum drugs and vice versa [50]. The hyper expression of EGFR, the hyperactivity of the MAP and Akt signaling pathways, the high frequency of DNA aberrations in combination with the disruption of DNA repair processes, the mutations of p53 and BRCA1 provide increased sensitivity to platinum preparations [46]. In many cisplatin-resistant cells of BC, an increased activity of topoisomerase II is observed [51]. The resistance to platinum is also indicated by the expression of LRP (lung resistance protein) [52]. Thus, using relatively inexpensive immunohistochemical studies, backing up their results with available blood PCR, buccal smear, etc., we are able to determine the optimal sensitivity of the tumor to cytostatic medicines and choose the optimal chemotherapy regimen. The results of the survey are shown in Table 1.

Table 1: The results of the review.

\begin{tabular}{|c|c|c|c|}
\hline Marker & Resistance & Toxicity & Antiestrogens Inhibitors of aromatase \\
\hline $\begin{array}{c}\text { Receptors of estrogen, } \\
\text { progesterone }\end{array}$ & & & Anthracyclines \\
\hline Her2/neu & Methotrexae Doxorubicin & Platinum \\
\hline Expression of EGFR & Methotrexate & Epirubicin Platinum \\
\hline Mutation of p53 & & & Taxanes \\
\hline Expression of RB1 & Platinum & & Anthracyclines \\
\hline Expression of Bcl2 & & & \\
\hline Expression of Top2A & Cyclophosphamide Platinum & & \\
\hline Expression of GST & & & \\
\hline
\end{tabular}




\begin{tabular}{|c|c|c|c|}
\hline Expression of P-gp & $\begin{array}{l}\text { Mitoxantrone Methotrexate Anthracyclines } \\
\text { Taxanes Winkaalkaloids }\end{array}$ & & Cyclophosphamide* \\
\hline Expression of TLE3 & & & Cyclophosphamide Taxanes \\
\hline $\begin{array}{l}\text { The absence or low expres- } \\
\text { sion of RFC }\end{array}$ & Methotrexate & & \\
\hline Polymorphism MTHFR & & Methotrexate & \\
\hline Expression of TS & Fluoropyrimidines & & \\
\hline $\begin{array}{l}\text { The missing or decreasing } \\
\text { function of DPD }\end{array}$ & & Fluoropyrimidines & \\
\hline Decreasing of TP & Capecitabine & & Fluorouracil \\
\hline BCRP & Winkaalkaloids & & \\
\hline MRP1 & $\begin{array}{cl}\text { Taxanes } & \text { Methotrexate Anthracyclines } \\
& \text { Winkaalkaloids }\end{array}$ & & \\
\hline $\begin{array}{l}\text { Expression of } \beta \text {-тубулінуIII } \\
\text { grade }\end{array}$ & & & $\begin{array}{l}\text { The combination of taxanes with cyclo- } \\
\text { phosphamide** } \\
\text { The combination of taxanes and anthracy- } \\
\text { clines }{ }^{* * *}\end{array}$ \\
\hline Mutation of BRCA1 & Winkaalkaloids & & Platinum \\
\hline The low expression of ERCC- 1 & & & Platinum \\
\hline Expression of LRP & $\mathrm{P}$ & & \\
\hline
\end{tabular}

\section{* does not affect on resistance}

** with positive estrogen receptors and elevated expression of marker

$* * *$ with the low expression of marker

\section{References}

1. Bagrova SG, Bychkov MB, Kuzminov AE, Mikhina ZP, Nasheletashvili DR (2014) Clinical recommendations for the diagnosis and treatment of patients with breast cancer, p. 43.

2. https://clinicaltrials.gov/

3. Tyulyandin SA (2000) Chemotherapy of disseminated breast cancer. Practical oncology 2: 3-11.

4. Buzdar AU, Ibrahim NK, Francis D, Booser DJ, Thomas ES, et al (2005) Significantly higher pathologic complete remission rate after neoadjuvant therapy with trastuzumab, paclitaxel, and epirubicin chemotherapy: results of a randomized trial in human epidermal growth factor receptor 2-positive operable breast cancer. J Clin Oncol 23(16): 3676-3685.

5. Buzdar AU, Valero V, Ibrahim NK, Francis D, Broglio KR, et al. (2007) Neoadjuvant therapy with paclitaxel followed by 5-fluorouracil, epirubicin, and cyclophosphamide chemotherapy and concurrent trastuzumab in human epidermal growth factor receptor-2 positive operable breast cancer: an update of the initial randomized study of patients with the same regimen. Clin Cancer Res 13(1): 228-233. 
6. NCCN Guidelines (2017) Invasive breast cancer.

7. Gershtein ES (2014) Growth factors, their receptors and underlying signaling proteins: from experiment to clinic. Progress in Molecular Oncology 1: 27-35.

8. Peredchikova NI, Stenina MB (2014) Drug therapy for breast cancer. Practice, Moscow, p. 284.

9. Novik AV (2011) The importance of biomarkers for diagnosing and determining the tactics of treatment of patients with tumors with unknown primary location. Practical oncology 12(4): 178-184.

10. http://vestnik.rncrr.ru/vestnik/v5/papers/litdobr_v5.htm

11. Inwald EC, Klinkhammer SM, Hofstädter F, Zeman F, Koller M, et al. (2013) Ki-67 is a prognostic parameter in breast cancer patients: results of a large population-based cohort of a cancer registry. Breast Cancer Res Treat 139(2): 539-552.

12. Kheirandish Sh, Fatemeh H (2015) Ki-67 protein: a proliferation index in breast cancer. Reviews Clin Medicine 2(4): 205-208.

13. Cuzick J, Dowsett M, Wale C, Salter J, Quinn E (2009) Prognostic value of acombined ER, PgR, Ki67, HER2 immunohistochemical (IHC4) score and the comparison with the GHI recurrence score-results from Trans ATAC. Cancer Res 69(24): 503.

14. Dowsett M, Smith IE, Ebbs SR, Dixon JM, Skene A, et al. (2005) Shortterm changes in Ki-67 during neoadjuvant treatment of primary breast cancer with anastrozole or tamoxifen alone or combined correlate with recurrence-free survival. Clin Cancer Res 11(2): 951-958.

15. Bondarenko IM, Zavizion VF, Artemenko MV (2017) Possibilities of immunohistochemical research in predicting the response to treatment and modification of treatment measures in lymphoma breast cancer (review of literature) Medical perspectives 22(4): 4-12.

16. Telegin LYu (2012) Pharmacogenetics of cyclophosphamide. INFRA Moscow, p. 81.

17. Brian Z Ring, Douglas TR, Robert SS, Rodney. A Beck TLE3 as a marker for chemotherapy.

18. Kostjuk SB (2013) The role of glutathione in the pathogenesis of breast Stransferazi iron. Proceedings of the National Academy of Sciences of Belarus. Series of Medical Sciences 1: 78-90.

19. Cao K, Stack DE, Ramanathan R, Gross ML, Rogan EG, et al. (1998) Synthesis and structure elucidation of estrogen quinones conjugated with cysteine, $\mathrm{N}$-acetylcysteine, and glutathione. Chem Res Toxicol 11(8): 909-916.

20. Demidchik YE, Kostiuk SA, Tretyak IY (2016) Mechanisms of cellular chemio resistance in breast cancer. The Belarus Eye, Minsk, Belarus, p. 152.

21. Nevozhai DV (2006) Current views on the mechanism of the antitumor effect of methotrexate and resistance to it. Pacific Medical Journal 4: 12 16.

22. Toffoli G, Russo A, Innocenti F, Corona G, Tumolo S, et al. (2003) Effect of methylene tetra hydrofolate reductase $677 \mathrm{C}-\mathrm{T}$ polymorphismontoxicity and homocysteine plasma level after chronic methotrexate treatment of ovarian cancer patients 103(3): 294-299.

23. Kuleva SA (2013) Delayed elimination of methotrexate after high-dose infusion. Vopr Onkol 59(1): 126-131.

24. Interpretatory NI (2015) Guidelines for chemotherapy of tumor diseases. Practical medicine, Moscow, p. 688.

25. Esentaeva CE (2013) Modern possibilities of chemotarget therapy for metastatic colorectal cancer. Oncology and radiology of Kazakhstan 2: 7-11.

26. https://oncohemakey.com/5-fluoropyrimidines/
27. Protsenko SA (2007) The search for ways to individualize antitumor therapy. Practical oncology 8(4): 173-181.

28. Szuławska A, Małgorzata C (2006) Molekularne mechanizmy działania antracyklin. Postepy Hig Med Dosw 60: 78-100.

29. Karen EO, Knudsen H, Birgitte BR, Balslev E, Knoop A, et al. (2004) Amplification of HER2 and TOP2A and deletion of TOP2A genes in breast cancer investigated by new FISH probes. Acta Oncologica 43(1): 35-42.

30. Nitiss L (2009) Targeting DNA topoisomerase II in cancer chemotherapy. Nat Rev Cancer 9(5): 338-350.

31. Choi YH, Yu AM (2014) ABC transporters in multidrug resistance and pharmacokinetics, and strategies for drug development. CurrPharm Des 20(5):793-807.

32. Qingcheng M, Unadkat JD (2015) Role of the breast cancer resistance protein (BCRP/ABCG2) in drug transport-an update. AAPSJ 17(1): 6582.

33. Ji YY, Qiong H, Youyun Y, Jian TZ, Mei ZZ, et al. (2009) Characterization and analyses of multi drug resistance-associated protein 1 (MRP1/ABCC1) polymorphisms in Chinese population. Pharmacogenet Genomics 19(3): 206-216.

34. Schepotin IB, Zotov AS, Lyubota RV, Anikusko NF, Love II (2012) Clinical significance of p53 mutations in breast cancer (review of literature). Clinical Oncology 8(4): 1-3.

35. Fojo T, Giannakakou P. Taxol and other microtubuline-interactive agents Current Opinion in Oncologic, Endocrine \& Metabolic Investigational Drugs. London, UK, pp. 293-304.

36. Yihong W, Joseph A, Sparano, Susan F, Lesley S, et al. (2013) High expression of class III $\beta$-tubulin predicts good response to neoadjuvant taxaneand doxorubicin/cyclophosphamide-based chemotherapy in estrogen receptor-negative breast cancer. Clin Breast Cancer 13(2): 103108.

37. Patrick Lebok, Melike Öztürk, Heilenkötter U, Jaenicke F, Müller V, et al (1987) High levels of class III $\beta$-tubulinex pression areas sociated with aggressive tumor features in breast cancer. Oncol Lett 11(3): 1987-1994.

38. Pentheroudakis G, Anna B, Konstantine TK, Ralf Kronenwett, Ralph MW, et al. (2011) Prognostic utility of $\beta$-tubulin isotype III and correlations with other molecular and clinicopathological variables in patients with early breast cancer: a translational Hellenic Cooperative Oncology Group (HeCOG) study. Breast Cancer Res Treat 127(1): 179-193.

39. Klimenko VV (2015) Molecular markers of the effectiveness of preoperative chemotherapy of locally advanced breast cancer.

40. Wang TH, Wang HS, Soong YK (2000) Paclitaxel-induced cell death: Where the cell cycle and apoptosis come together. Cancer. 88(11): 26192628.

41. Gasparini G, Caffo O, Barni S, Frontini L, Testolin A, et al. (1994) Vinorelbine is an active antiproliferative agent in pretreated advanced breast cancer patients: a phase II study. J Clin Oncol 12(10): 2094-2101.

42. Jordan MA, Thrower D, Wilson L (1991) Mechanism of inhibition of cell proliferation by Vinca alkaloids. Cancer Res Apr 51(8): 2212-2222.

43. Kamath K, Wilson L, Cabral F, Jordan M (2005) Beta III tubulin induce spaclitaxel resistance in association with reduced effects on microtubule dynamic instability. J Biol Chem 280(13): 12902-12907.

44. Stavrovskaya AA, Gens GP (2015) New in the study of multiple drug resistance of breast cancer cells. Advances in Molecular Oncology. 1: 3951.

45. Colinr J, Jennifere Q, Paulb M, Patrickg J, Paulharkin D, et al. (2007) BRCA1, a potential predictive biomarker in the treatment of breast cancer. The Oncologist 12(2): 142-150. 
46. Nakanishi T, Ross DD (2012) Breast cancer resistance protein (BCRP/ ABCG2): its role in multidrug resistance and regulation of its gene expression. Chin J Cancer 31(2): 73-99.

47. Albert B, Denisa I, Helena P (2013) Vincristine as an inductor of drug resistance marker expression in neoplastic cells/ in vincristine. In: Coello JM, Yo Sabres D (Eds.), Nova Science Publishers, New York, USA, pp. 1-31.

48. https://www.rlsnet.ru/mnn_index_id_512.htm

49. Novik AV, Zhabin AS (2011) The importance of biomarkers for diagnosing and determining the tactics of treatment of patients with tumors with unknown primary localization. Practical oncology 12(4): 178-184.
50. Domagala P, Jakubowska A, Jaworska BK, Kaczmarek K, Durda K, et al. (2015) Prevalence of germline mutations in genes engaged in dna damage repair by homologous recombination in patients with triplenegative and hereditary non-triple-negative breast cancers. PLoS One 10(6): e0130393.

51. Chekhun VF, Shishova YV (2000) Modern views on mechanisms of formation of drug resistance of tumors. Oncology 2(1-2): 11-15.

52. Blokhin DY (2009) Mechanisms of formation of drug resistance of tumor cells. Clinical oncohematology 2(1): 167-175.

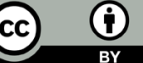

Creative Commons Attribution 4.0 International License

For possible submissions Click Here

\section{Submit Article}

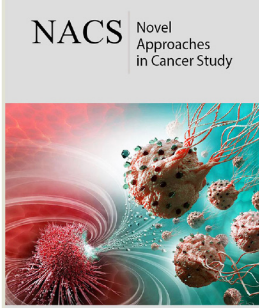

\section{Novel Approaches in Cancer Study}

\section{Benefits of Publishing with us}

- High-level peer review and editorial services

- Freely accessible online immediately upon publication

- Authors retain the copyright to their work

- Licensing it under a Creative Commons license

- Visibility through different online platforms 